\title{
Integration of active specific immunotherapy and cell therapy into the protocols of biomedical management of interstitial pneumonia with autoimmune features $-A$ case study
}

\author{
Roni Moya $^{1,3}$, Mike KS Chan ${ }^{2}$, Michelle BF Wong ${ }^{3}$ and Dmytro Klokol ${ }^{1,3 *}$ \\ ${ }^{1}$ European Wellness Academy, Asia-Pacific \\ ${ }^{2}$ FCTI, EU \\ ${ }^{3}$ International University of BioRegenerative Sciences, USA
}

\begin{abstract}
Non-specific interstitial pneumonia (NSIP) is the second most common morphological and pathological pattern of interstitial lung diseases. A significant proportion of patients with interstitial lung disease (ILD) manifest autoimmune features consisting, among others, of a morphologic domain with multi-compartment involvement and specific autoantibodies, as well as association to other autoimmune pathologies, such as systemic lupus erythematous, rheumatoid arthritis and Hashimoto thyroiditis. The symptoms of non-specific interstitial include insidious onset of dyspnea and dry cough with a restrictive pattern of decreased lung function and reduced gas exchange capacity. Corticosteroids, anti-fibrotics and immunosuppressants are the classical protocol to treat the condition, but management should be carefully individualized due to the wide heterogeneity of IPAF and lack of evidence in this particular subgroup of patients. The multidisciplinary immunological approach with xenogeneic and/or autologous peptides and its derived immune extracts might be a way to induce autoimmune tolerance, based on the anergy mechanism of secretion of immunomodulatory cytokines, such as IL-10 and transforming growth factor- $\beta$ (TGF- $\beta$ ) and T-regulatory cells. Here we report a clinical case of 47 years old woman with IPAF and organized pneumonia $(\mathrm{OP})$ with rheumatic complications addressed integrativelly with the autologous Active Specific Immunotherapy (ASI), xenogeneic peptide immunotherapy, ozone autohemotherapy and nutritional antioxidants perfusion. The integrative biomedical program was run from July 2019 until February 2020 with stabilization of lung degeneration and compliances, consequent modulation of chronic inflammation, progressive reduction of dry cough and joint pain, less fatigue, better sleep quality, overall energy and further decreasing of pharmaceutical's dosage. This article opens a scientific discussion on how to address positively to chronic autoimmune conditions within the use of biomedical multifunctional and natural tools like autologous and xenogeneic immunotherapy in combination with a more conservative pharmaceutical protocol.
\end{abstract}

\begin{abstract}
Abbreviations: ASI: Active Specific Immunotherapy; APCs: Antigen-presenting Cells (APCs); CTD: Connective Tissue Diseases; HLA: Human Leukocyte Antigen; ILD: Interstitial Lung Disease: IPAF: Interstitial Pneumonia with Autoimmune Features; LAK: LymphokineActivated Killer; MHC: Major Histocompatibility Complex; NSIP: Non-Specific Interstitial Pneumonia; OP: Organized Pneumonia; Th3: T-helper type 3; TGF- $\beta$ : Transforming Growth Factor- $\beta$; TNF: Tumor Necrosis Factor.
\end{abstract}

\section{Introduction}

Non-specific interstitial pneumonia (NSIP) is the second most common morphological and pathological pattern of interstitial lung diseases [1]. The Interstitial pneumonia with autoimmune features (IPAF) has been internationally defined to characterize interstitial lung disease (ILD), mainly associated with systemic sclerosis, rheumatoid arthritis, inflammatory myopathies (dermatomyositis, polymyositis), Sjogren syndrome, systemic lupus erythematous, and mixed connective tissue diseases (CTD) [2]. Worldwide experts from different medical specialties have conceptualized this state as an undifferentiated CTDILD, lung-dominant CTD, and autoimmune-featured ILD, using different but overlapping criteria and terminology [3]. The symptoms of non-specific interstitial pneumonia are elusive and by definition non-specific, including insidious onset of dyspnea and dry cough with a restrictive pattern of decreased lung function and reduced gas exchange capacity. Prognosis is generally intermediate between that of idiopathic pulmonary fibrosis and connective tissue disease-associated interstitial lung disease, but substantially variable according to the predominant histologic and radiologic patterns [4]. The prevalence of IPAF varies between 7 and $34 \%$ of all ILDs depending mainly on the population studied with the mean age from 60 to 65 years, with balanced gender, although some studies reported a younger mean age ( $\sim 55$ years) and a predominance of white non-smoking women. More than $90 \%$ of patients with IPAF have at least one of the serological criteria $[5,6]$ (Ahmad K, 2017); (Oldham JM, 2016). Apart of the histological and CT imaging findings, the serological diagnostic point to the main positive auto-antibody showed in several series such as Anti-SSa, anti-

${ }^{*}$ Correspondence to: Dmytro Klokol, International University of BioRegenerative Sciences, USA; E-mail: Dr.dmytro@iubrs.com

Key words: active specific immunotherapy, interstitial lung disease, interstitial pneumonia with autoimmune features, xenogeneic peptides

Received: October 05, 2020; Accepted: October 30, 2020; Published: November 09, 2020 
synthetase antibodies, as well as high titer rheumatoid factor [5,6]. The disease management is still inconclusive with data regarding IPAF treatment only limited to case series and further research needed to determine the optimal treatment strategy in the IPAF population. Even though, corticosteroids, anti-fibrotic drugs and immunosuppressive agents are considered as the mainstay of treatment [7].

\section{Methodology}

\section{Active Specific Immunotherapy (ASI)}

The defining characteristic of an autoimmune disease is the existence of T- and B-cell auto reactivity directed against self-proteins auto-antigens [8]. Immunotherapy is based on the modulation of the immune system divided into specific, targeting direct response against accurate antigens (ex. peptide therapy and dendritic cell therapy), or nonspecific, boosting the general immunity without aiming any particular biomarker (cytokine therapy and lymphokine-activated killer (LAK) cell therapy) [9]. In order to be successful, the optimal protective immune system must balance between elimination of foreign pathogens and tolerance of self-antigens. To prevent any unwanted damage caused by activated $\mathrm{T}$ cells to surrounding tissues, the immune system has evolved a variety of mechanisms, or 'check points' that are used to modulate the duration and amplitude of the immune response. This dampens the immune response and protects against damage because of inflammation and autoimmunity [10]. Immune tolerance therefore is the key point to trigger an effective approach to autoimmunity and the activation of regulatory $\mathrm{T}$ cells will exert it effects through the secretion of immunomodulatory cytokines, such as IL-10 and transforming growth factor- $\beta$ (TGF- $\beta$ ) $[11,12]$. The regulatory cells, sometimes referred to as T-helper type 3 (Th3) cells have the potential to home to the target tissue of autoimmune disease driven by their specificity for auto antigen available at this site, and once stimulated, to inhibit the entire autoimmune response by the local release of cytokines 'bystander suppression' $[11,13]$. The regulatory population is currently being defined by expression of CD4, CD25 and CTLA-4 [14]. Thus, the Active Specific Immunotherapy (ASI) is an autologous individualized immunotherapy, able to induce CD4+ CD25+ cells with immuneregulatory properties, aiming to stimulate the modulatory cascade of T-regulatory cells, inducible by self-patient's cytokines and autoantigen peptides administration and hence developing self-tolerance to hyper-reactivity $[8,15]$.

\section{ASI preparation}

After three days clotting, $30 \mathrm{ml}$ of the buffy-coat of the patient's peripheral blood was isolated under the GMP laminar-flow-technique and the immune molecular elements were separated by different patented biochemical and physical steps, followed by proprietary oxygenation biotherapy [15]. Afterwards a culture of Immune activating additives substances was then added to the buffy-coat to prepare 30 vials of vaccine (1.1 $\mathrm{ml}$ each for subcutaneous injections).

\section{ASI protocol}

From July 2019 until October 2019, 30 vials of ASI vaccines were administered subcutaneously three times per week for a total of 10 weeks.

\section{Immunomodulatory peptide's transfer factors}

The mainstream approach to IPAF rests among anti-fibrotic and immunosuppressant drugs such as azathioprine, mycophenolate mofetil, corticosteroids, cyclophosphamide and rituximab (anti-B-cell CD20 monoclonal antibody) [4]. The clinician should be reminded however of the general detrimental effect of corticosteroid and/or immunosuppressive therapy in patients with IPAF and be careful with the known unfortunate side effects and toxicity. The most attractive strategy therefore may be the targeted induction of regulatory cell populations, which will be favored by using relatively low doses of highly soluble peptides/antigens. Peptides are short chains of 20 or less amino acids that can induce or inhibit biological effects. It can control gene expression and protein synthesis and preventing changes that cause aging and disease. As resultant, the innovation of peptides therapy offers a regulatory mechanism to trigger the natural immunity control [16]. Henry Lawrence showed years ago that delayed cutaneous hyper-reactivity could be modulated by soluble immune extracts of leukocytes called transfer factors molecules [17]. Recently, is suggested that the peptide's transfer factors soluble molecules are able to induce CD4+ CD25+ cells with immune regulatory properties and therefore promote the generation of Th3-like regulatory $\mathrm{T}$ cells, including with the absence of any adjuvant, with the administration by a tolerogenic route (e.g. oral or intranasal) [18]. This combination of factors implies that the nature of the antigen-presenting cells (APCs) involved in T-cell activation may be critical: the avoidance of inflammatory stimuli that might activate APCs or the targeting of tolerance-promoting APCs at mucosal sites both favor the generation of regulatory cells [19]. Oral peptides immunotherapy approach has several advantages when compared to the whole antigen application. Peptides can be produced in large quantities and in highly purified forms much more readily than whole recombinant antigens. Peptide sequences can be selected to ensure solubility and to target specific disease-associated human leukocyte antigen (HLA) molecules. Using peptides avoids the risk of dose-limiting biological side effects since peptides do not retain the bioactivity of the intact molecule [8]. In summary, peptide's immunotherapy is focused on biochemical and molecular managing of cellular immune-tolerance with a specific selected group of nano-size low weight molecules $(<3 \mathrm{~nm}$ and $<3000 \mathrm{Da})$ that can better and faster be processed by the APCs, which enhances its the optimal bioavailability, i.e. how effectively the antigen or peptide can be delivered to the sites of greatest potential effectiveness.

\section{Peptide's preparation}

The nano-size peptides $(<3 \mathrm{~nm}$ and $<3000 \mathrm{Da})$ use the property technology of specific peptides derived from activated leucocyte lysates from European certified rabbits serving as vectors to boost the antigen-specific clonal expansion of immune cells and immune tolerance. The mechanism is explained as follows: Once the rabbit's leucocytes are infected by an intra-cellular antigen, it will initiate the innate phagocytosis which will signaling the activation of the natural killers and killers factors, as well as the Th1-CD4, T-CD8 key stimulatory cytokines (e.g., TNF, INF, IL2, etc.) and T-regulatory immunosuppressive molecules (e.g., IL-10). The phagocytes and lymphocytes cell-mediated signalling molecules will therefore setup the specific B-Cells and B-regulatory antibodies factors production. All these signalling resultants are called immunity transfer factors and can be purified in vitro into nano-size peptides to serve as adaptive immunotherapeutic modulatory treatment against chronic inflammation and hyperactive autoantibodies. In summary, these cells can inhibit auto antigens specific helper $\mathrm{T}$ cells, which secrete proinflammatory cytokines and are responsible for immune reactions in autoimmune diseases such as IPAF.

\section{Organ specific cell therapy}

The mechanisms of organ-specific cell therapy are based on "targeting" the specific organ ("homing effect") and replacement of 
impaired cell pool and activation/stimulation of the specific cellular factors [20]. The same applies to other types of molecules (especially of low molecules mass), which are responsible for the controlled mechanisms in all single cell and their inter-relationships with cells of other organs and tissues [20]. Xenogeneic cell therapy is an implantation of specific organic bioactive peptides, vitamins, minerals and growth factors to nourish living tissues, inducing immune tolerance and stimulating cellular renewal [21]. The extracts with bioactive components are from closed colonies, which are heavily monitored and its biological subtracts completely purified in order to eliminate any risk of genetic diseases, unwanted pathological or hyperactivity sensitiveness conditions and can safely be applied for medical and nutritional purposes.

Several books and studies support the use of cellular factors that are able of breaking down into enzymes, polypeptides, deoxyribonucleic acids, ribonucleic acids and other basic organic substances, recognizing the target specific host's molecular receptors and by that, expected to stimulate the growth and function of existing tissue, awakening of dormant cells and repairing old or malfunctioning cells [22]. The high amounts of peptides and proteins as cell materials are ensured zero denaturation to preserve the cell structure and keep the highest biological potency retained through cryopreservation at $-152^{\circ} \mathrm{C}$ to $-196^{\circ} \mathrm{C}$ and sterilization with property's infusion technology.

\section{Case Study}

In June 2018, a non-smoker, afro-descendent, 47 years old woman presented herself at the Luanda's Hospital, Angola, with symptoms of acute respiratory allergy, severe pulmonary insufficiency and left lung inflammation. Her clinical history didn't include any previous allergic, infectious or other chronic conditions. After an inconclusive diagnostic, she was treated with classical anti-allergic drugs and antibiotics and released from the hospital. By October 2018, she proceeded with chronic cough and swollen joints with rigidity and pain, being given corticosteroids and more antibiotics with no further definitive diagnostic. In December 2018, the patient noticed a progressive dry cough, loss of weight, fatigue, respiratory complications and joint pain and decided to have a full check-up in a private hospital in Brazil, being diagnosed with advanced state of rheumatoid arthritis, with prednisolone $60 \mathrm{mg}$ as treatment; which improved the inflammation symptoms but worsened her cough. In February 2019, she was received at the Angola's hospital with a thromboembolism episode with pulmonary insufficiency complications. The CT scan from May 2019 showed bronchiectasis compatible with organized pneumonia (OP) aspects and non-specific interstitial pneumonia, being considered for a potential lung transplantation surgery. The definitive medication protocol was thus prednisolone $5 \mathrm{mg}$, azathioprine $150 \mathrm{mg}$, rivaroxaban $15 \mathrm{mg}$, olmesartan $20 \mathrm{mg}$, amlodipine $5 \mathrm{mg}$ and hydrochlorothiazide $12.5 \mathrm{mg}$. While on hold for the lung transplantation final decision, she kept with symptoms of fatigue, respiratory insufficiency compliance and chronic constant dry cough. On July 2019, she began a protocol of ozone autohemotherapy with $70 \mathrm{mcg} / 200 \mathrm{ml}$ twice a week that slightly improved her condition and delayed the transplantation procedure.

In order to recovery the lung status, reduce the acute autoimmune inflammatory relapses, respiratory deficiency condition and extreme fatigue, during this period of time and until February 2020, our biomedical team started an integrative natural immunomodulation and regenerative approach divided in two phases: 1 - Active Specific Immunotherapy (ASI) with immune peptides transfer factors, together with intravenous nutritional supplements; 2 - Intravenous nutritional supplements and organ-specific cell therapy. The aim of this program was to highlight the natural integrative immunomodulatory and regenerative perspectives in order to balance de overall healthy wellbeing status, avoid chronic lung degeneration leading to transplantation, auto reactiveness and further chronic inflammatory associated complications.

From July 2019 until October 2019, the patient received $2.5 \mathrm{ml}$ vials of $1200 \mathrm{mg}$ nano size immune peptides and factors, given sublingually, three times per week. From December 2019 until February 2020, 9 vials with $5 \mathrm{ml}$ of frozen specific organic extracts were applied intramuscularly: 6 vials of lungs extracts; 1 vial of placenta, 1 vial of mesenchyme and 1 vial of thymus.

\section{Intravenous nutritional supplementation protocol}

Due to the poor metabolic and extreme fatigue condition, the nutritional intravenous perfusions were prescribed as follows.

One session of intravenous infusion per week for the first 4 weeks and therefore and until the end of the full program, every 15 days; starting on December 10 $0^{\text {th }}, 2019$.

\section{Infusion contents}

- Vitamin C $25 \mathrm{~g}$

- Alpha Lipoic Acid 600 mg

- Glutathione $600 \mathrm{mg}$

- B-Complex

- Minerals (Mg, Mn, Zn, Se)

- Amino acids pool

No changes at the prescribed conventional/pharmacological treatment were made during the integrative biomedical intervention period.

\section{Results}

On December 2018, after the proper diagnosis of IPAF with the related chronic complications and furthermore, the thrombotic episode on February 2019, the subject searched for the biomedical regenerative program, starting on June 2019. Patient's inflammatory chronicity with severe respiratory insufficiency, lack of cardiopulmonary debit compliance, plus the prescribed pharmaceutical toxicity had also been associated to the symptoms and signs of a progressive loss of weight, reduced appetite, gastric and intestinal malabsorption sensitiveness, insomnia (due to the incessant dry cough), extreme fatigue and nutritional deficiencies. The CT scan from May 2019, comparative to other previous from September 2018 showed overlapping expression of the interstitial changes that mainly concerned the pulmonary bases, comprising parenchymal densification with condensing internal traction bronchiectasis; compatible with aspects of organized pneumonia (OP) and concomitant NSIP. The weekly ozone autohemotherapy $70 \mathrm{mcg} / 200 \mathrm{ml}$ had already being in course before we met the patient and continued all along, within the hospital's doctor's described conventional pharmaceutical schedule. From July 2019 until October 2019, the 30 vials of $1.1 \mathrm{ml}, 3 \mathrm{x}$ week of the ASI vaccine was administered subcutaneously within the $2.5 \mathrm{ml}$ of the nano immune modulators peptides factors, given sublingually. From December 2019 until February 2020, 9 vials with $5 \mathrm{ml}$ of sheep's frozen specific organic extracts were applied intramuscularly: 6 vials of lungs extracts; 1 vial of placenta, 1 vial of mesenchyme and 1 vial of thymus. By November 
2019, patient presented no respiratory infectious relapses, no pain and less tiredness. The chronic cough episodes were reduced and the sleep quality was improved. Even tough, the biochemical analysis from November 2019 presented slightly anemia $\mathrm{Hb}(11.1 \mathrm{mg} / \mathrm{dl})$ with lymphocytosis, hyperglycemia $(111 \mathrm{mg} / \mathrm{dl})$, elevation of the creatinine $(1.10 \mathrm{mg} / \mathrm{dl})$, hypercholesterolemia $(283 \mathrm{mg} / \mathrm{dl})$ and reducing in the overall vitamins and minerals. Despite of being already under ozone autohemotherapy, which is a valuable clinical tool to reduce inflammation, prevent infections and boost the oxygenation [23], it was then decided to support patient's poor metabolic and nutritional status with nutritional intravenous cocktail perfusions addressed once a week or every two weeks until the end of the biomedical program. On January 2020, the blood test profile had no nutritional deficiencies and the hemoglobin levels reached the normal range of $12.0 \mathrm{mg} / \mathrm{dl}$. The cholesterol was $252 \mathrm{mg} / \mathrm{dl}$ but the triglycerides reduced from $115 \mathrm{mg} /$ $\mathrm{dl}$ to $83 \mathrm{mg} / \mathrm{dl}$. Glycaemia continued at $111 \mathrm{mg} / \mathrm{dl}$. The comparative CT scan at the same date concluded the stability of interstitial changes, compatible with NSIP associated with OP, but with no worsening either progression to connective chronic fibrosis, adenopathies or vascular complications. The continuous dry cough was practically absent as much as the energy and sleep quality was recovery. The patient began with a light physical activity rehabilitation program with no respiratory severe constrains. The lung transplantation schedule was definitively of the board and the corticoids/immune suppressors from the hospital's protocol reviewed to be progressively reduced.

\section{Discussion}

Non-specific interstitial pneumonia typically tends to present in middle-aged adults, 40-50 years of age. It may be common in Caucasian-European populations [1]. Overall prevalence is higher in women due to high association with collagen vascular diseases, but the prevalence of idiopathic NSIP is similar in both genders [1]. We presented a case of an afro-descendent, 47 years old woman suffering from an interstitial pneumonia with autoimmune features (IPAF) and organized pneumonia (OP), whose main symptoms were severe fatigue due to the respiratory insufficiency, joint pain and constant dry cough; medicated with the standard corticoids and immunosuppressants for this condition. The first integrative protocol to enhance her life-quality was the ozone autohemotherapy. In short, Ozone therapy causes an increase in the red blood cell glycolysis rate leading to the stimulation of 2,3-diphosphoglycerate and an increase in the ATP, glutathione peroxidase, catalase and superoxide dismutase antioxidants enzymes [24]. In the lung, it significantly increases mean airway and specific airway resistance without changing dynamic or static pulmonary compliance or viscous or elastic work. It also significantly reduces maximal trans pulmonary pressure, increases respiratory rate and decreases tidal volume [25]. Even with a weekly ozone therapy, the patient continued to feel her difficulties, but slowly showed an overall better disposition. After July 2019, the biomedical regenerative program began and ASI vaccine with peptide's immunomodulatory transfer factors initiated. ASI is a specific autologous immunotherapy based on the context of auto anti-idiotypic antibodies as regulatory agents to induce a proper immune anergy, defined as antigen-specific nonresponsiveness to hyperactivity autoimmune responses. It is already clear that in terms of peptides, the same peptide that activates immune response can induce anergy trough the major histocompatibility complex (MHC) class I, i.e. anergy induction in CD8 T cells. To the down-regulation of auto reactive anti-idiotypic antibodies by anergy to be maintained, the continued presence of the antigen is required. In the absence of antigen, hypo-responsiveness may be relatively short-lived (a matter of several weeks) but can be kept by chronic administration of antigen (e.g. by weekly injections), which acts to sustain peripheral tolerance very effectively [26-28]. The ASI and the molecular immune peptide's transfer factors, the first administrated subcutaneously and the second orally may act together at the MHC receptors cascade triggering the tolerance of autoimmunity by the t-regulatory cells, Interleukin-10, tumor necrosis factor (TNF) and consequently auto-antibodies death by apoptotic pathway mediated by the Fas/Fas ligand (FasL) [29]. From December 2019 until February 2020 the xenogeneic frozen cells therapy had its course, being applied intramuscularly together with the intravenous nutrient's perfusions. As the cryogenic technology preserves the cell's best specific enzymatic and molecular regenerative potential, the previous standardized laboratory purification of the extracts allowed the xenogeneic molecules and peptides to keep the immune tolerance anergy and at the same time, boosting the lungs recovery; in this particular case, by prevention of more damaging and hopefully by future restoring the best possible cardiopulmonary function. The positive and interesting result from the frozen cells approach tough was that no allergic, toxic or acute inflammatory responses were noticed. On the contrary, specifically after the first week of the first intramuscular application, patient showed a very good well-being disposition and almost no dry cough at all, which allowed her doctors to cancel the transplantation schedule and progressively reduce the pharmaceuticals; leading obviously to less systemic immune and physical over-suppression. The antioxidant perfusions had either its role not just by replacing the nutrients deficiency and detoxify by enhancing the endogenous antioxidants enzymes (e.g., catalase, superoxide dismutase, glutathione peroxidase, etc.), but also by modulate the chronically stressed and suppressed hypothalamicpituitary axis under corticoids effects. It's related that high dosages of vitamin $\mathrm{C}$ can modulate the cortisol by the adrenocorticotrophic receptors, acting as a natural adaptogen [30-32]. It's comprehensive the limits of this article with just one particular case with IPAF related where integrative protocol of several approaches took place and one cannot certify exactly which individual technic had better effect; but this was never the point. The aim was to address the bunch of personalized natural regenerative biomedical treatments available under the most scientifically rigor, mainly on the immunological background to help a suffering autoimmune patient, already condemned to a life-care immunosuppressant and transplantation procedure and no further hope from any innovative conventional treatment. First, the biomedical program proved to do no harm. Afterwards it did not avoid the benefits of the pharmaceuticals already in use (on the contrary; after the biomedical protocol, doctors started to reduce its dosages). Secondly, the CT scan stabilized, showing no further damaging. Thirdly, exhaustive tiredness, chronic cough, extreme pain and psychological and physical inability were specifically improved upon the beginning of the program (which were not before, while just under the pharmacological approach). Last but not least, the immunity status improved as well, showing not so ever any infective relapses and no need for antibiotics. It's understood that more studies must be developed taken in consideration the highest level of technology and scientifically knowledge available in order to open new doors to the integrative biomedical field.

\section{Conclusion}

The active specific immunotherapy, associated with Immunomodulatory peptide's factors, organ-specific cryogenically preserved cell's extracts, ozone autohemotherapy and nutritional perfusions might induce the tolerance anergy in the interstitial pneumonia with autoimmune features and organized pneumonia, 
Moya R (2020) Integration of active specific immunotherapy and cell therapy into the protocols of biomedical management of interstitial pneumonia with autoimmune features - A case study

have no negative side-effects, do not interfere with the benefits of the conventional drugs, improve the immunity against relapsing infections and help to reduce the negative associated symptoms to the condition, either psychological or physical, opening new perspectives on science to address chronic and degenerative pathologies.

\section{References}

1. Craig Hacking YW (2020) Non-specific interstitial pneumonia. Radiopaedia.

2. Fischer A, du Bois R (2012) Interstitial lung disease in connective tissue disorders. Lancet 380: 689-698. [Crossref]

3. Cottin V (2006) Interstitial lung disease: are we missing formes frustes of connective tissue disease? Eur Respir J 28: 893-896. [Crossref]

4. Fernandes L, Nasser M, Ahmad K, Cottin V (2019) Interstitial Pneumonia with Autoimmune Features (IPAF). Front Med (Lausanne) 6: 209. [Crossref]

5. Ahmad K, Barba T, Gamondes D, Ginoux M, Khouatra C, et al. (2017) Interstitial pneumonia with autoimmune features: clinical, radiologic, and histological characteristics and outcome in a series of 57 patients. Respir Med 123: 56-62. [Crossref]

6. Oldham JM, Adegunsoye A, Valenzi E, Lee C, Witt L, et al. (2016) Characterisation of patients with interstitial pneumonia with autoimmune features. Eur Respir J 47: 1767-1775. [Crossref]

7. Mathai SC, Danoff SK (2016) Management of interstitial lung disease associated with connective tissue disease. $B M J 352$ : h6819. [Crossref]

8. Peakman M, Dayan CM (2001) Antigen-specific immunotherapy for autoimmune disease: fighting fire with fire? Immunology 104: 361-366. [Crossref]

9. Blomberg OS, Spagnuolo L, de Visser KE (2018) Immune regulation of metastasis: Mechanistic insights and therapeutic opportunities. Dis Model Mech. [Crossref]

10. Steven A, Fisher SA, Robinson BW (2016) Immunotherapy for Lung Cancer. Respirology 21: 821-833. [Crossref]

11. Burkhart C, Liu GY, Anderton SM, Metzler B, Wraith DC (1999) Peptide-induced T cell regulation of experimental autoimmune encephalomyelitis: a role for IL-10. Int Immunol 11: 1625-1634. [Crossref]

12. Sakaguchi S, Sakaguchi N (2000) Regulatory T cells: key controllers of immunologic. Cell 101: 455-458. [Crossref]

13. Homann D, Holz A, Bot A, Coon B, Wolfe T, et al. (1999). Autoreactive CD4+ T cells protect from autoimmune diabetes via bystander suppression using the IL-4/Stat6 pathway. Immunity 11: 463-472. [Crossref]

14. Roncarolo MG, Levings MK (2000) The role of different subsets of $\mathrm{T}$ regulatory cells in controlling autoimmunity. Curr Opin Immunol 12: 676-683. [Crossref]

15. Roni Moya, Chan MKS, Klokol D, Pan SY (2018) Active Specific Immunotherapy (ASI) and Gcmaf Forte in Management of Metastatic Invasive Carcinoma-Overview of the Therapeutic Modalities and A Case Report. J Clin Exp Immunol 3: 1-4.
16. Cobb LJ, Lee C, Xiao J, Yen K, Wong RG, et al. (2016) Naturally occurring mitochondrial-derived peptides are age- dependent regulators of apoptosis, insulin sensitivity, and inflammatory markers. Aging 8: 796-809. [Crossref]

17. Lawrence HS (1955) The transfer in humans delayed skin sensitivity to streptococcal M substance and to tuberculin with disrupted leukocytes. J Clin Invest 34: 209. [Crossref]

18. Anderton S, Burkhart C, Metzler B, Wraith D (1999) Mechanisms of central and peripheral T-cell tolerance: lessons from experimental models of multiple sclerosis. Immunol Rev 169: 123-137. [Crossref]

19. Sallusto F, Lanzavecchia A (1999) Mobilizing dendritic cells for tolerance, priming, and chronic inflammation. J Exp Med 189: 611-614. [Crossref]

20. Klokol D, Nallenthiran L, Nalapko Y, Chernykh V, Yefimov S, et al.(2020) Treatment strategies in management of chronic kidney disease from perspective of biological regenerative medicine. J Stem Cell Res Ther 6: 1-9.

21. Hayashi M (2006) Development of new therapies, including regeneration of the kidney, for chronic kidney diseases. Clin Exp Nephrol 10: 99-101. [Crossref]

22. Klokol D, Chan MKS (2019) Stem Cells in regenerative medicine: Carpe diem, carpe vitum. UK: Troubador; $495 \mathrm{pp}$.

23. Elvis AM, Ekta JS (2011) Ozone therapy: A clinical review. J Nat Sci Biol Med 2: 66-70. [Crossref]

24. Sunnen GV (2014) SARS and ozone therapy: Theoretical considerations.

25. Viebahn-Hänsler R (2003) The use of ozone in medicine: Mechanisms of action.

26. Rocha B, Tanchot C, Von Boehmer H (1993) Clonal anergy blocks in vivo growth of mature $\mathrm{T}$ cells and can be reversed in the absence of antigen. $J$ Exp Med 177: 15171521. [Crossref]

27. Alferink J, Schittek B, Schönrich G, Hämmerling GJ, Arnold B (1995) Long life span of tolerant $\mathrm{T}$ cells and the role of antigen in maintenance of peripheral tolerance. Int Immunol 7: 331-336. [Crossref]

28. Bercovici N, Delon J, Cambouris C, Escriou N, Debré P, et al. (1999) Chronic intravenous injections of antigen induce and maintain tolerance in $\mathrm{T}$ cell receptortransgenic mice. Eur J Immunol 29: 345-354. [Crossref]

29. Sytwu HK, Liblau RS, McDevitt HO (1996) The roles of Fas/APO-1 +CD95) and TNF in antigen-induced programmed cell death in T cell receptor transgenic mice. Immunity 5: 17-30. [Crossref]

30. Padayatty SJ, Doppman JL, Chang R, Wang Y, Gill J, et al. (2007) Human adrenal glands secrete vitamin $\mathrm{C}$ in response to adrenocorticotrophic hormone. Am J Clin Nutr 86: 145-149. [Crossref]

31. Brody S, Preut R, Schommer K, Schürmeyer TH (2002) A randomized controlled trial of high dose ascorbic acid for reduction of blood pressure, cortisol, and subjective responses to psychological stress. Psychopharmacology 159: 319-324. [Crossref]

32. Hooper MH, Carr A, Marik PE (2019) The adrenal-vitamin C axis: from fish to guinea pigs and primates. Crit Care 23: 29. [Crossref]

Copyright: (C2020 Moya R. This is an open-access article distributed under the terms of the Creative Commons Attribution License, which permits unrestricted use, distribution, and reproduction in any medium, provided the original author and source are credited. 